\title{
PARTICLE PRODUCTION AND CORRELATIONS IN HADRON-HADRON COLLISIONS IN THE OUAL MONTE-CARLO CHAIN FRAGMENTATION MODEL
}

\author{
J. Ranft * \\ CERN, Geneva \\ and \\ S. Ritter \\ Karl-Marx-Universität Leipzig
}

A B S T R A C T

We study a dual Monte-Carlo two-chain fragmentation model for hadron-hadron collisions. A comparison of the model with data from proton-proton, pion-proton and kaon-proton interactions is presented. This includes inclusive $x$-distributions in the fragmentation region, resonance production and forward-backward correlations.

(To be published in $\mathrm{Z}$. Physik C)

*) Permanent address: Karl-Marx-Universität, Leipzig, GDR 


\section{INTRODUCTION}

Particle production in high-energy hadron-hadron and hadronnucleus collisions is actively studied at present, but serious predictions from quantum chromodynamics (QCD) are lacking. Therefore different models are used to interpret the data. Models such as the one we consider here are constructed in the framework of the parton model together with dynamical assumptions motivated by QCD. We study the multi-chain fragmentation model, which starts from the dual topological unitarization (DTU) scheme [1]. Capella and Tran Thanh Van developed a two-chain model for hadron-hadron interactions [2] on the basis of the DTU scheme and generalized it to a multi-chain model for hadron-nucleus interactions [3]. This is the approach we already followed in Ref. [4]. There we presented a two-chain Monte-Carlo fragmentation model for hadron-hadron interactions and a multi-chain Monte-Carlo fragmentation model for hadron-nucleus collisions. The two-chain model for hadron-hadron scattering processes has the following features:

(i) We construct two parton chains built up only from valence quarks of the projectile and the target particle.

(ii) Going into the centre of mass system of the chains we fragment them into hadrons or hadron resonances using a Monte-Carlo chain decay fragmentation model developed earlier by the authors [5-7]. This model has been tested in comparison with data from $e^{+} e^{-}$annihilation [6] and from lepton-hadron interactions [8]. In order to apply such a hadronization scheme also to soft hadron-hadron interactions, we have to make the basic assumption that quark and antiquark jets from $e^{+} e^{-}$annihilation or diquark jets from lepton-hadron scattering processes do not differ strongly from the ones produced in hadron-hadron collisions. This jet universality is not derived theoretically, but experimental data strongly suggest such an assumption, see [9] for a review.

(iii) For chains of very low invariant mass we slightly modify the kinematics and create directly stable hadrons or hadron resonances. 
(iv) Transforming all created hadrons and hadron resonances back into the laboratory system, we obtain a complete final hadronic state.

Since we use a Monte-Carlo method we are able to generate exclusive events conserving exactly energy momentum and all additive quantum numbers.

(v) The subsequent decay of hadron resonances into stable final hadrons is realized using the Monte-Carlo code DECAY [10].

Since we are only interested in multiparticle production in the energy range from 5 or $10 \mathrm{GeV}$ up to $1 \mathrm{TeV}$, it is sufficient to consider two chains containing only the valence quarks in hadron-hadron collisions. This is not correct for larger energies, i.e. at collider energies. We then have to add further chains involving also sea quarks [11] according to the AGK rules [12]. Such an extension of the model has already been discussed in Ref. [13].

Our multi-chain model for hadron-nucleus interactions $[4,14]$ and nucleus-nucleus interactions [15] is an extension of the two-chain model for hadron-hadron collisions. These multi-chain models are only expected to describe data, provided the two-chain model works well for hadron-hadron interactions.

The contents of the paper are as follows: In section 2 we give a brief description of the underlying Monte-Carlo procedure. A more detailed description is given in Ref. [4]. In sections 3 and 4 we present inclusive Feynman $x$-distributions in the fragmentation region and resonance production. Finally forward-backward correlations are discussed in section 5 .

\section{FORMULATION OF THE MODEL}

Describing hadron-scattering processes in the framework of the two-chain fragmentation model, it is assumed that the interaction separates the valence quarks of each incident hadron into two coloured systems, which give rise to two multiparticle chains [2]. In Fig. 1a, $b$ and $c$ all possible two-chain systems for baryon-baryon, antibaryonbaryon and meson-baryon interactions are shown. 
The energy or momentum fraction $x_{q}$ carried by the single valence quark is given by the distribution

$$
d_{B}^{\text {val }}(x) \sim \frac{(1-x)^{b}}{x^{1 / 2}}
$$

with $b=2$ for baryons and $b=1.5$ for mesons. The values of the parameter $b$ used here differ from that given in Ref. [4]. This leads to a better fit to the inclusive distributions in the fragmentation region. In the case of baryons the dressed diquark gets the remaining fraction $x_{d}=1-x_{q}$. Usually we get $x_{d}>x_{q}$.

In the case of mesons we have two possibilities. Either the quark gets an $x_{q}$-fraction according to distribution [2] and the antiquark obtains the remaining energy $x_{\bar{q}}=1-x_{q^{\prime}}$ or vice versa. As already mentioned in the introduction, building up the chains only from valence quarks is not correct at larger energies but is a good approximation at laboratory energies below $1 \mathrm{TeV}$.

In the ISR energy range $E_{\text {lab }} \approx 0.5-2.0 \mathrm{TeV}$ we get, by including the additional chains containing sea quarks only, a rise of the average charged multiplicity of $6 \pm 1$ per cent.

Knowing the $x$-fractions of the partons and the centre of mass energy of the collision we can easily calculate the invariant masses of the chains. The subsequent transition into hadrons is treated separately for each chain in its centre of mass system on the basis of a Monte-Carlo chain decay fragmentation model [5-7]. For very low energy chains, i.e. chains with invariant masses below approximately $1-1.5 \mathrm{GeV} / \mathrm{c}^{2}$ depending on the quantum numbers, we correct the kinematics and create directly a hadron resonance or a stable hadron constructed from the initial quarks of the chain.

An additional Lorentz boost of each chain back into the laboratory system provides a complete final hadronic state produced in a hadron-hadron interaction. The decay of hadron resonances into stable hadrons is realized using the Monte-Carlo code DECAY [10]. Since our Monte-Carlo chain decay model exactly conserves energy momentum and additive quantum numbers for each chain, we obtain a 
final multiparticle state conserving these quantities as well. So our Monte-Carlo model is especially well suited to study exclusive quantities and can be used as an event generator.

\section{THE ERAGMENTATION REGION}

In this chapter we compare our Monte-Carlo results for inclusive $x$-distributions $x(d o / d x)$ in the laboratory system and the centre of mass system to data from $\mathrm{K}^{-} \mathrm{p}$ and $\mathrm{pp}$ interactions $[17,18]$.

In Fig. $2 a, b$ and $c$ we plot the inclusive cross-section $x(d \sigma / d x)$ for the process $\mathrm{K}^{-}+\rightarrow^{+}+\mathrm{X}$ in the centre of mass system for the target fragmentation region. The energy dependence of $\pi^{+}$and $\pi^{-}$ production (see Fig. $2 a$ and $b$ ) as well as the difference between $\pi^{+}$ and $\pi^{-}$distributions (see Fig. 2 c) is well described by our two-chain Monte-Carlo model.

In Fig. 3 we show the inclusive longitudinal momentum distribution for pp-scattering processes into charged pions, charged kaons, protons and antiprotons in the laboratory frame at $\mathrm{p}_{\mathrm{lab}}=175 \mathrm{GeV} / \mathrm{c}$. We get a reasonably good agreement to the data [18] in the region $x \leqslant 0.6$. For larger $x$-values a comparison with data is problematic for three reasons. At first we have not included diffractive particle production in the model so we are not able to describe effects caused by diffractively produced protons. Secondly there are uncertainties in the data points at $x$-values larger than $x \approx 0.6$ in the case of charged protons, charged kaons and antiprotons because model assumptions are used concerning the $p_{\mathcal{L}}$-behaviour at large $x$ (see Ref. [18]).

The third point is that our Monte-Carlo statistics for $\mathrm{K}^{+}, \pi^{+}$and p production is limited although we use 40000 events.

\section{RESONANCE PRODUCTION}

We consider average multiplicities and multiplicity ratios as a function of the centre of mass energy $\sqrt{s}$ or as a function of the transverse momentum for several resonances produced in pp or $\pi^{+} p$ interactions. Furthermore we present the inclusive cross-section $x(d \sigma / d x)$ for $e^{\circ}$ production in $k^{-} p$ scattering processes. The results of 
our Monte-Carlo two-chain model (almost 5000 events per energy) are compared to recent data measured at the ISR at CERN $[17,19,20]$.

In Table 1 we compare the average multiplicity ratios $\left\langle e^{\infty}\right\rangle /\left\langle\pi^{-}\right\rangle$, $\left\langle\bar{K}^{* 0}\right\rangle /\left\langle\mathrm{K}^{-}\right\rangle,\left\langle\mathrm{K}^{* 0}\right\rangle /\left\langle\mathrm{K}^{+}\right\rangle$and $\langle\phi\rangle /\left\langle\mathrm{e}^{0}\right\rangle$ measured in pp-collisions at $S_{s}=63 \mathrm{GeV}[19]$ with results of our Monte-Carlo two-chain model.

Furthermore we show in Figs. 4,5 and 6 the $\sqrt{s}$-dependence for the multiplicity ratios $\left.\langle\phi\rangle / \rho^{0}\right\rangle$ and $\left\langle\rho^{0}\right\rangle /\left\langle\pi^{-}\right\rangle$as well as the $p_{\perp}$-dependence of the ratio $\left\langle\rho^{0}\right\rangle /\left\langle\pi^{-}\right\rangle$at $\sqrt{s}=63 \mathrm{GeV}$.

In all three cases we get a reasonably good agreement with the data [19], almost within the data errors. In Fig. 7 we plot average $Q^{\circ}$-multiplicities versus the squared centre of mass energy for $\pi^{+} p$ and $\pi^{-} p$ interactions and obtain also a good agreement with data presented in Ref. [20].

The inclusive $x$-distribution $x(d \sigma / d x)$ of $e^{0}$ produced in $\mathrm{K}^{-} \mathrm{p}$ reactions at $\sqrt{s}=32 \mathrm{GeV}$ is given in Fig. 8 . In the considered $x$-region $x \leqslant 0$ our two-chain model agrees well with the data [17].

Finally, in Fig. 9 we plot model predictions for average multiplicities of several resonances as a function of the laboratory momentum in pp-collisions.

\section{FORWARD-BACKWARD CORRELATIONS}

Long-range correlations between particles produced at large distances in rapidity may give valuable information about the multiparticle production mechanism. Here we consider in our model the correlation between the multiplicity of particles emitted into one centre of mass hemisphere, and the multiplicity in the opposite hemischere (forward-backward multiplicity correlations) first studied in Ref. [21].

In Fig. 10 we show average forward multiplicities $\left\langle n_{F}\right.$ 〉 versus the backward multiplicity $n_{B}$ of proton-proton interactions at three different centre of mass energies. The Monte-Carlo results of our two-chain model are compared to data presented in Ref. [20]. 
In Fig. 11 we have plotted average backward multiplicity at $\sqrt{s}=13.7 \mathrm{GeV}$. The results of our two-chain model are compared to data [20] measured in $\pi^{-} p$ collisions.

In both cases we obtain a reasonably good agreement with the data.

It is however to be noted that a part of the measured forwardbackward correlations actually results from short-range correlations. The slope of the forward-backward correlation in the model drops considerably if we exclude short-range correlations by considering only particles with $|y| \geqslant 0.5$ or 1 .

\section{SUMMARY}

The formulation of the two-chain model on the basis of a Monte-Carlo method allows the calculation of inclusive as well as exclusive quantities. The model is well suited to generate exclusive events conserving energy momentum and all additive quantum numbers.

Continuing our studies of this model [4], the data on inclusive $x$-distributions are compared here with resonance production and forward-backward multiplicity correlations measured in $p p, \pi p$ and $k p$ interactions. In general we get a good agreement with the data.

Since we have not taken into account diffractive events, our model is not able to describe leading particle effects at Feynman $x_{F}$ values close to $x_{F}=1$.

\section{ACKNOWLEDGEMENTS}

One of the authors (J.R.) thanks Dr. K. Goebel and the Radiation Protection Group of CERN for the possibility to perform a large part of the work reported during a stay at CERN. Furthermore we thank P. Aurenche and J. Tran Than Van for useful discussions. 


\section{REFERENCES}

1. - G. Veneziano, Nucl. Phys. B74 (1974) and B117 (1976) 519;

- G. Chew and C.Rosensweig, Nucl.Phys. B104 (1976) 290, Phys. Rep. 41C (1978) 263.

2. - A. Capella, U. Sukhatme, C.I.Tan and J.Tran Than Van, Phys. Lett. $\underline{81 \mathrm{~B}}(1980)$;

- A. Capella, U. Sukhatme and J. Tran Than Van, Z. Physik C3 (1980) 329.

3. A. Capella and J. Tran Than Van, Phys. Lett. 93B (1980) 146 and 2. Physik C10 (1981) 249.

4. J. Ranft and S. Ritter, Z. Physik C20 (1983) 347.

5. J. Ranft and S. Ritter, Acta Phys. Pol. B11 (1980) 259.

6. S. Ritter, 2. Physik c16 (1982) 27.

7. S. Ritter, Description of the Monte-Carlo code BAMJET, Comp. Phys. Comm. - 31 (1984) 393.

8. S. Ritter, unpublished.

9. K. Fialkowaki and W. Kittel, Re. Progr. Phys. $\underline{46}$ (1983) 1283.

10. K. HäuBgen and S. Ritter, Descreiption of the Monte-Carlo code DECAY, Comp. Phys. Comm. 31 (1984) 411.

11. - A.B. Kaidalov and K.A. Ter-Martirosyan, Phys. Lett. 117B (1982) 247;

- P. Aurenche and F.W. Bopp, Phys. Lett. 114B (1982) 363;

- A. Capella and J. Tran Thanh Van, Phys. Lett. 114B (1982) 450, Z. Physik C23 (1984) 165 and orsay preprint LPTHE $83 / 10$.

12. V.A. Abramavoski, V.N. Gribov and D.V. Kancheli, Yad. Fiz. 18 (1971) 595.

13. - P. Aurenche, F.W. Bopp and J. Ranft, Annecy preprint LAPP$\mathrm{TH}-83$ (1983) to appear in 2. Physik $\mathrm{C}$;

- P. Aurenche, F.W. Bopp and J. Ranft, Annecy preprint LAPP$\mathrm{TH}-95$ (1983);

- J. Ranft, P. Aurenche and F.W. Bopp, CERN preprint TH 3775 (1983) to appear in Z. Physik C.

14. J. Ranft and S. Ritter, CERN preprint CERN- TIS-RP/128/PP (1984), submitted to $Z$. Physik $C$.

15. H.-J. Möhring, J. Ranft and S. Ritter, to be submitted to 2. Physik C.

16. T. Akeson et al., Axial Fieeld Spectrometer Collaborations, CERN preprint CERN-EP/83-75 (1983). 
17. R. Göttgens et al., CERN preprint CERN-EP/83-161 (1983).

18. A.E. Brenner et al., Phys. Rev. D27 (1983) 2580.

19. C.W. Fabjan et al., Nucl. Phys. B203 (1982) 27.

20. A. Wroblewski, Review talk at the XIV Int. Symposium on Multiparticle Dynamics, Granlibakhen, Lake Tahoe, USA, June 1983.

21. A. Capella and J. Tran Thanh Van, Z. Physik, C18 (1983) 85. 
TABLE 1

Average multiplicity $\left.\operatorname{ratios}\left\langle\mathrm{e}^{0}\right\rangle /\left\langle\pi^{-}\right\rangle,\left\langle\overrightarrow{\mathrm{K}}^{* 0}\right\rangle / \mathrm{K}^{-}\right\rangle$and $\langle\phi\rangle /\left\langle e^{0}\right\rangle$ in pp-collisions.

The Monte-Carlo results are compared to data measured at $\sqrt{s}=63 \mathrm{GeV}[19]$.

\begin{tabular}{ccc} 
Exp. [19] & $\begin{array}{c}\text { Monte-Carlo results } \\
\sqrt{s}=69.31 \mathrm{GeV}\end{array}$ \\
\hline $\mathrm{s}=63 \mathrm{GeV}$ & 0.160 \\
$\left\langle\mathrm{Q}^{0}\right\rangle /\left\langle\pi^{-}\right\rangle$ & $0.2 \pm 0.03 \pm 0.04$ & 0.440 \\
$\left\langle\overline{\mathrm{K}}^{* 0}\right\rangle /\left\langle\mathrm{K}^{-}\right\rangle$ & $0.64 \pm 0.09 \pm 0.03$ & 0.425 \\
$\left\langle\mathrm{~K}^{* 0}\right\rangle /\left\langle\mathrm{K}^{+}\right\rangle$ & $0.55 \pm 0-08 \pm 0.03$ & 0.072 \\
$\langle\phi\rangle /\left\langle\mathrm{Q}^{0}\right\rangle$ & $0.09 \pm 0.02 \pm 0.02$ & \\
\hline
\end{tabular}




\section{FIGURE CAPTIONS}

Fig. 1. All possible diagrams for two-chain systems in
(a) baryon-baryon,
(b) antibaryon-baryon and
(c) meson-baryon interactions.

$B, \bar{B}, M$ stand for baryon, antibaryon and meson; $\mathrm{x}^{\mathrm{q}}$ and $\mathrm{x}^{\mathrm{d}}$ are the quark and diquark momentum fractions, respectively. The indices $\mathrm{P}$ and $\mathrm{T}$ stand for projectile and target particle.

Fig. 2. Inclusive cross-section $x \frac{d o}{d x}(n b)$ plotted in the centre of mass system for the processes
(a) $\mathrm{K}^{-}+\mathrm{p} \rightarrow \pi^{+}+\mathrm{X}$ at $\sqrt{\mathrm{s}}=110 \mathrm{GeV}$ and $\sqrt{\mathrm{s}}=70 \mathrm{GeV}$;
(b) $\mathrm{K}^{-}+\mathrm{p} \rightarrow \pi^{-}+\mathrm{X}$ at $\sqrt{\mathrm{s}}=110 \mathrm{GeV}$ and $\sqrt{\mathrm{s}}=70 \mathrm{GeV}$;
(c) $K^{-}+p \rightarrow \pi^{+}+x$ at $\sqrt{s}=110 \mathrm{GeV}$ and $\sqrt{s}=70 \mathrm{GeV}$.

The Monte-Carlo results (histograms) are compared to data from Ref. [17].

Fig. 3. Inclusive cross-section $x \frac{d \sigma}{d x}(m b)$ for $p \bar{p}$ interactions into charged pions, charged kaons, protons and antiprotons at $\mathrm{p}_{1 \mathrm{ab}}=175 \mathrm{GeV} / \mathrm{c}$ plotted in the laboratory system. The Monte-Carlo results (histograms) are compared with data from Ref. [18].

Fig. 4. Js-dependence of the average multiplicity ratio $\langle\phi\rangle /\left\langle\mathrm{e}^{c}\right\rangle$ for pp interactions. The Monte-Carlo results, represented by crosses, are compared with data from Ref. [19].

Fig. 5. The same quantity as in Fig. 4 but for the ratio $\left\langle 0^{-}\right\rangle /\left\langle\pi^{-}\right\rangle$.

Fig. 6. $\mathrm{p}_{\perp}$-dependence of the average multiplicity ratio $\left\langle e^{\circ}\right\rangle /\left\langle\pi^{-}\right\rangle$for pp interactions. The Monte-Carlo results (histograms) are compared with data from Ref. [19] measured at $\sqrt{s}=63 \mathrm{GeV}$. 
Fig. 7. Average multiplicities for (a) $\pi^{\circ}$, (b) $e^{0}$ versus the squared centre of mass energy $s$ measured in $\pi^{+} p$ and $\pi^{-} p$ reactions. The Monte-Carlo results are compared to data from Ref. [20].

Fig. 8. The inclusive cross-section $x \frac{d \sigma}{d x}$ for the process $\mathrm{K}^{-}+\mathrm{p}+\mathrm{e}^{0}+\mathrm{x}$ at $\sqrt{\mathrm{s}}=32 \mathrm{GeV}$ plotted in the centre of mass system. The Monte-Carlo results (histograms) are compared to data from Ref. [17].

Fig. 9. Predictions of our two-chain Monte-Carlo model for the $\mathrm{p}_{1 \mathrm{ab}}$-dependence of average multiplicities for several resonances.

Fig. 10. $\left\langle n_{F}\right\rangle$ as a function of $n_{B}$ in pp-collisions at different $\mathrm{CM}$ energies. The Monte-Carlo results are compared with data presented in Ref. [20].

Fig. 11. $\left\langle n_{B}\right\rangle$ as a function of $n_{F}$ in $\pi^{-} p$ collisions at $V_{s}=13.7 \mathrm{GeV}$. The Monte-Carlo results are compared with data presented in Ref. [20]. 


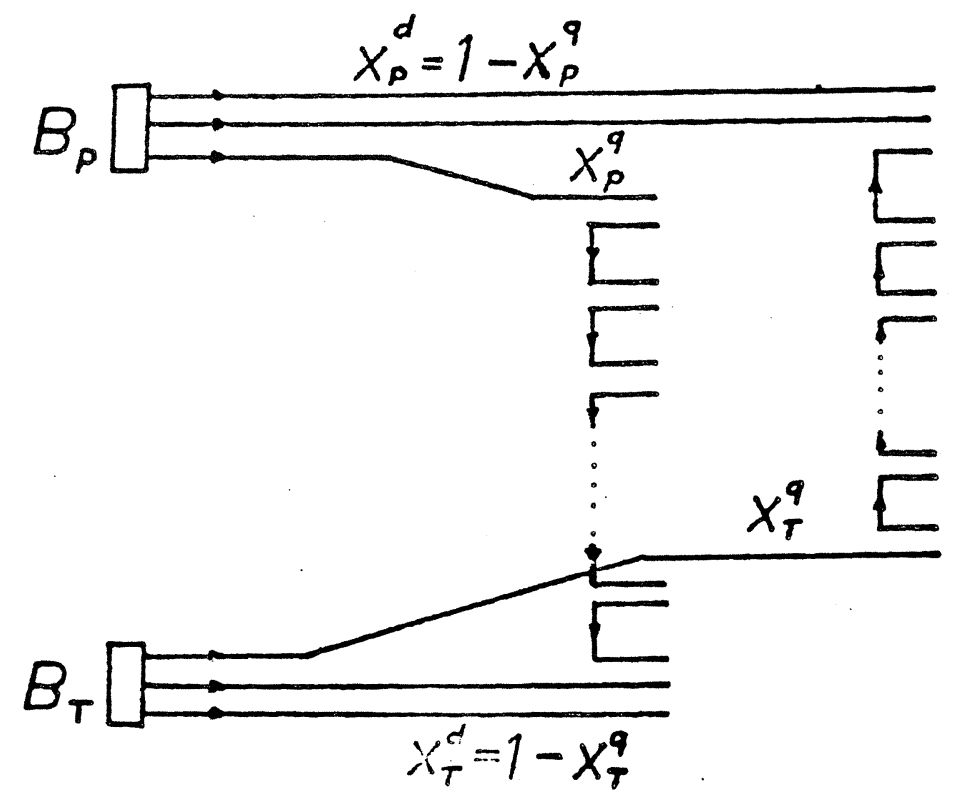

(a)

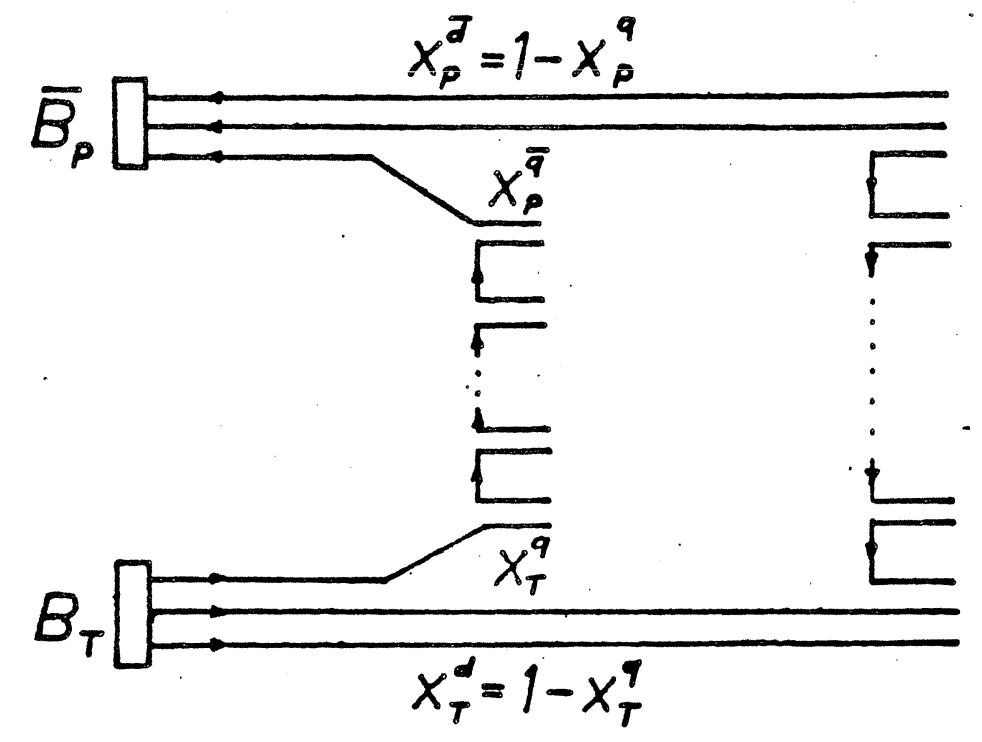

(b)
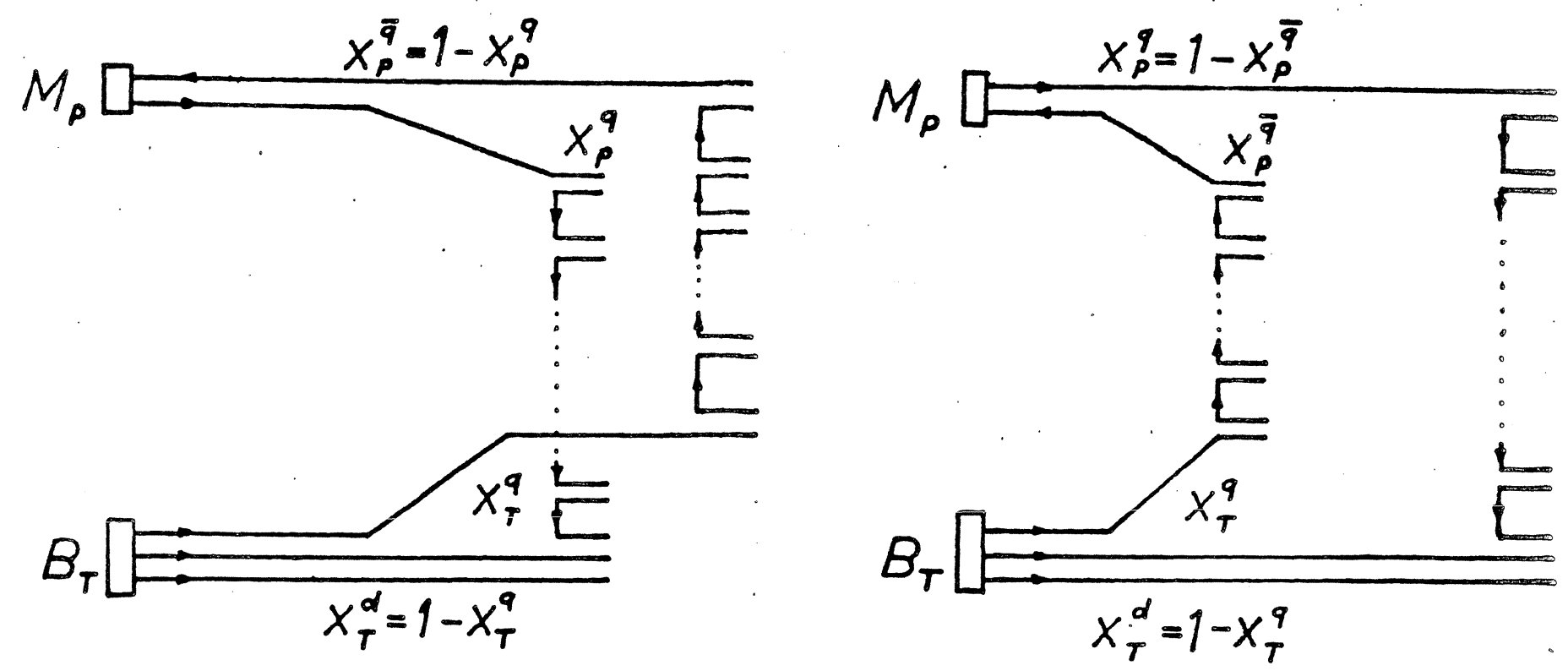

(c)

$F / G .1$ 
$x \frac{d \sigma}{d x}(\mu b)$
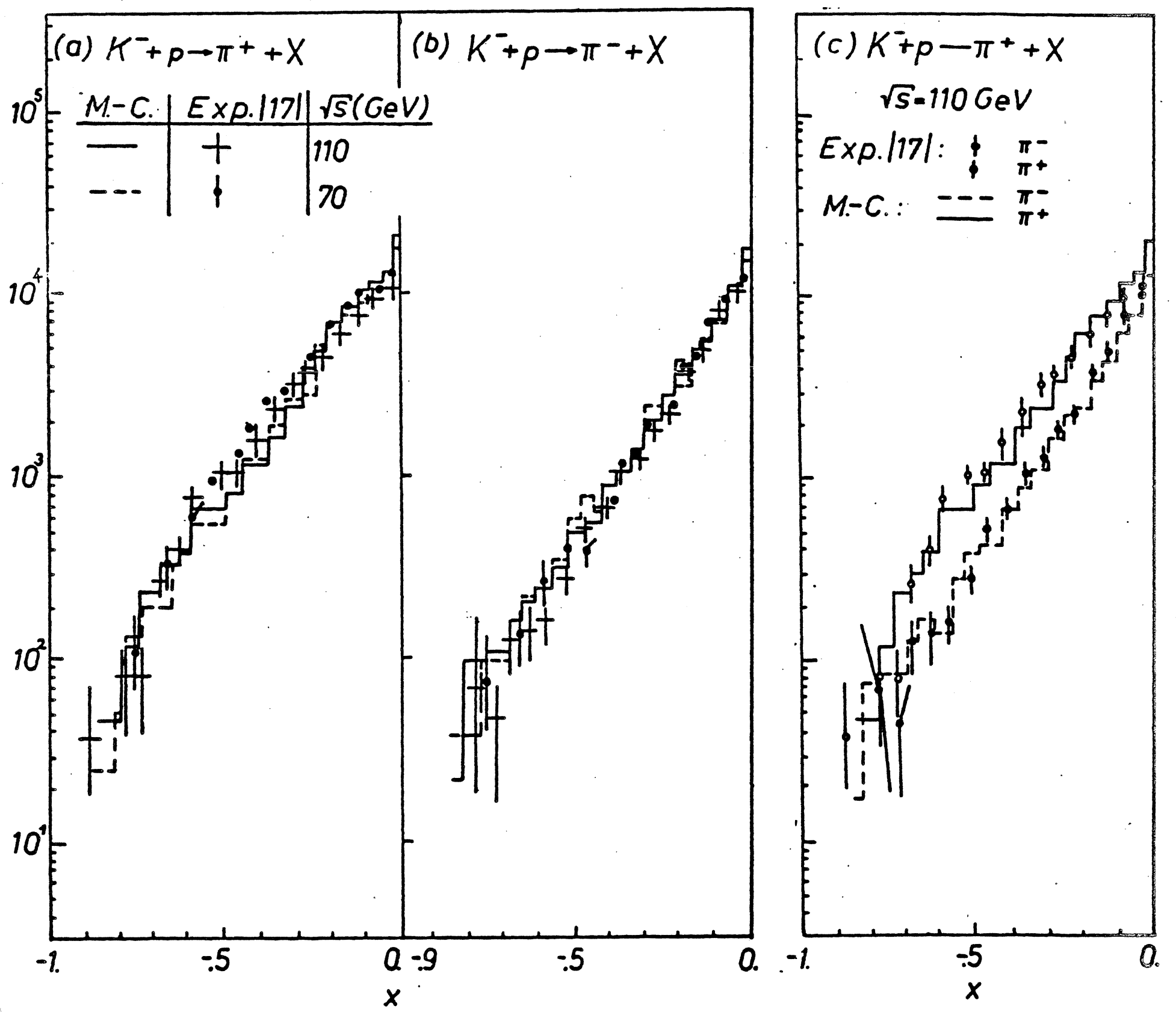

F/G. 2 


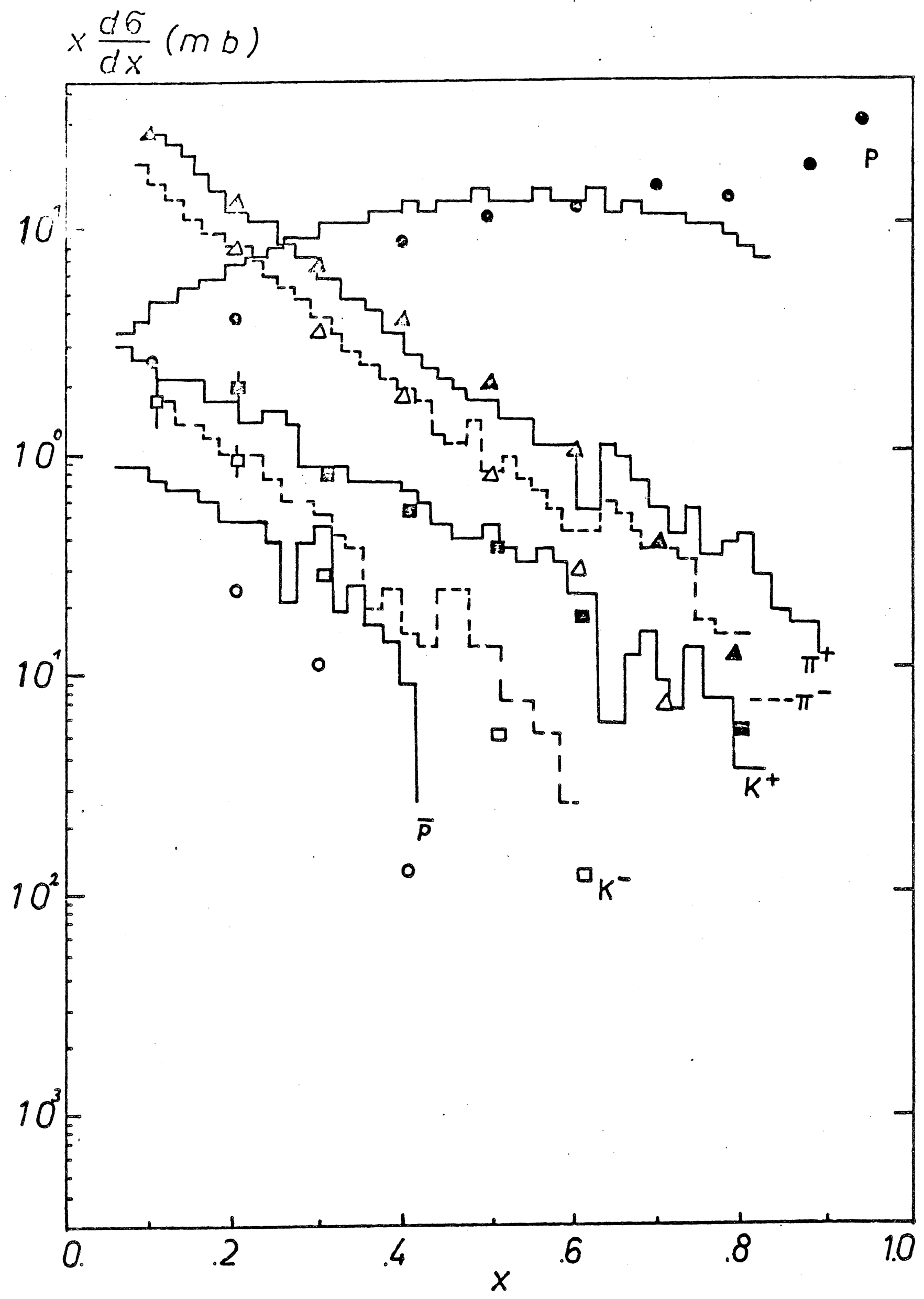

FIG.3 


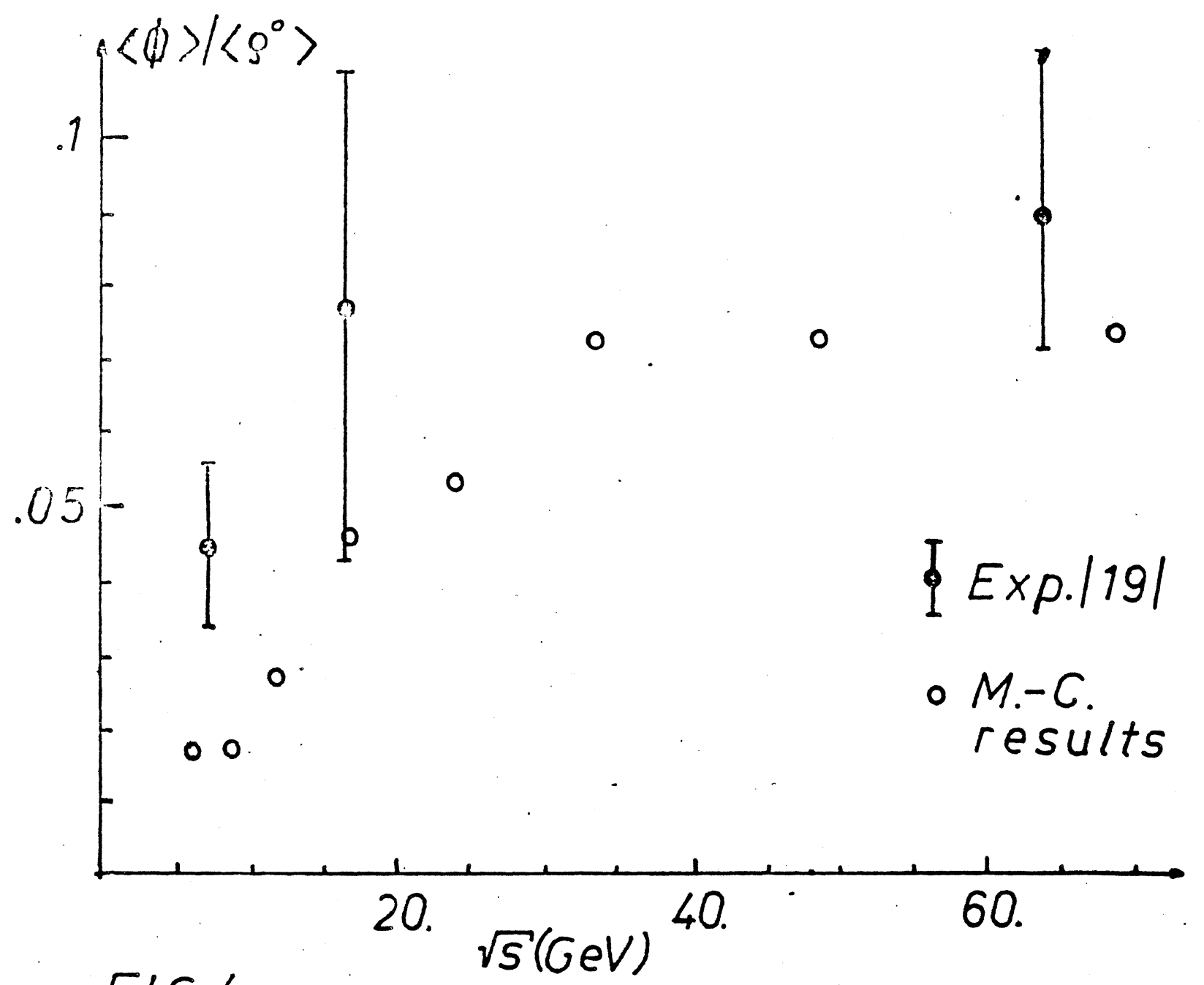

FIG.4

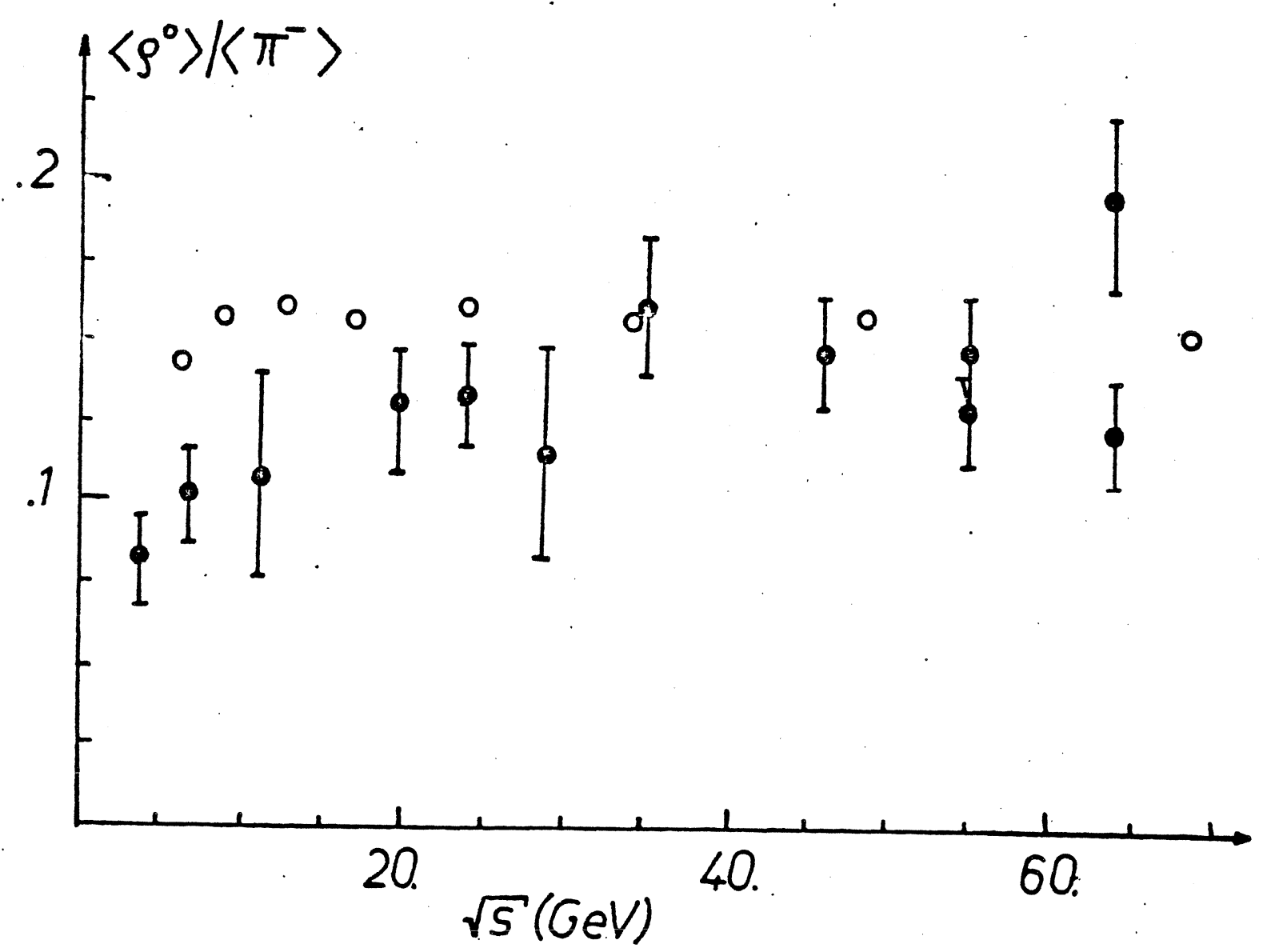

FIG.5 


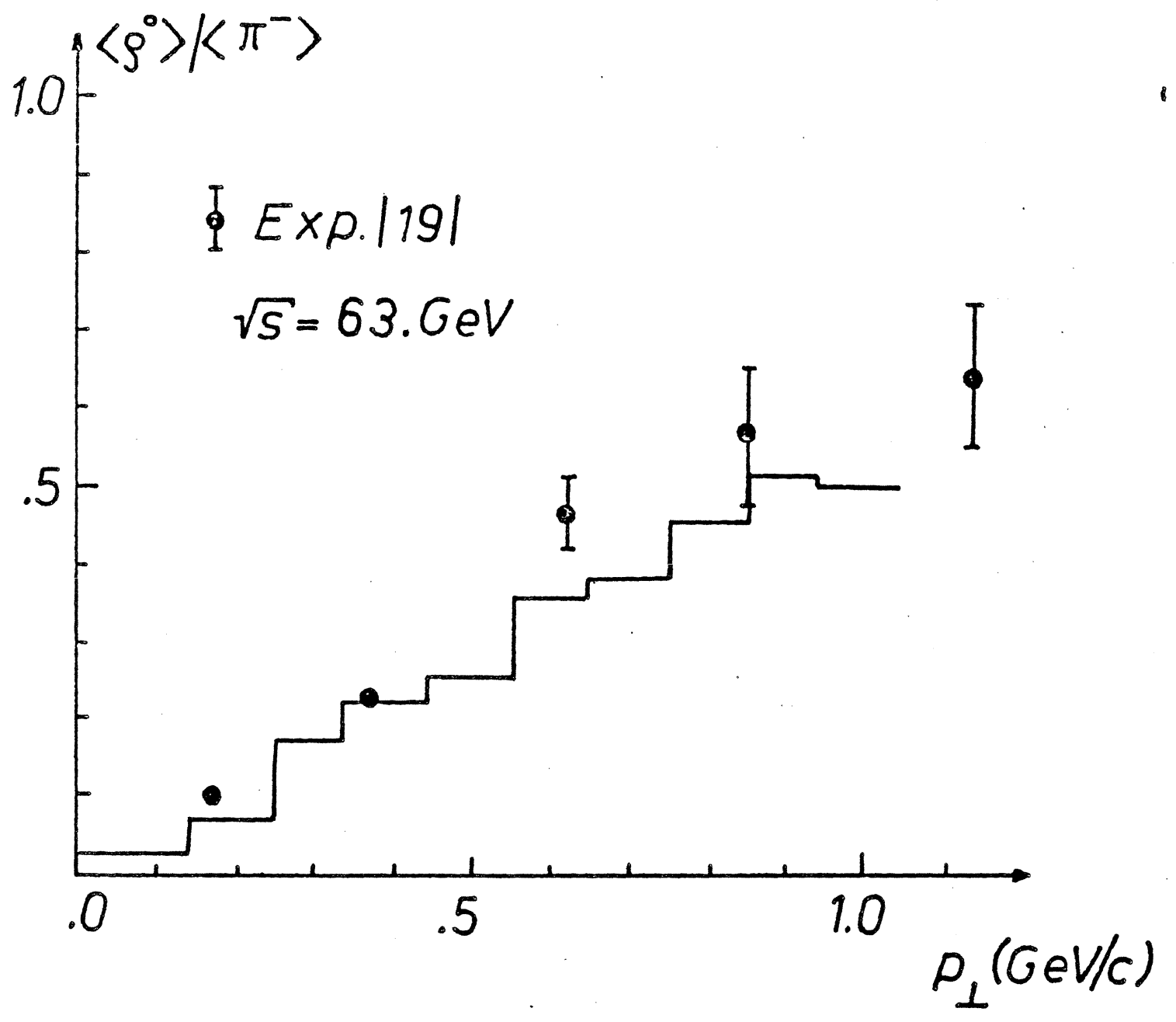

FIG. 6 


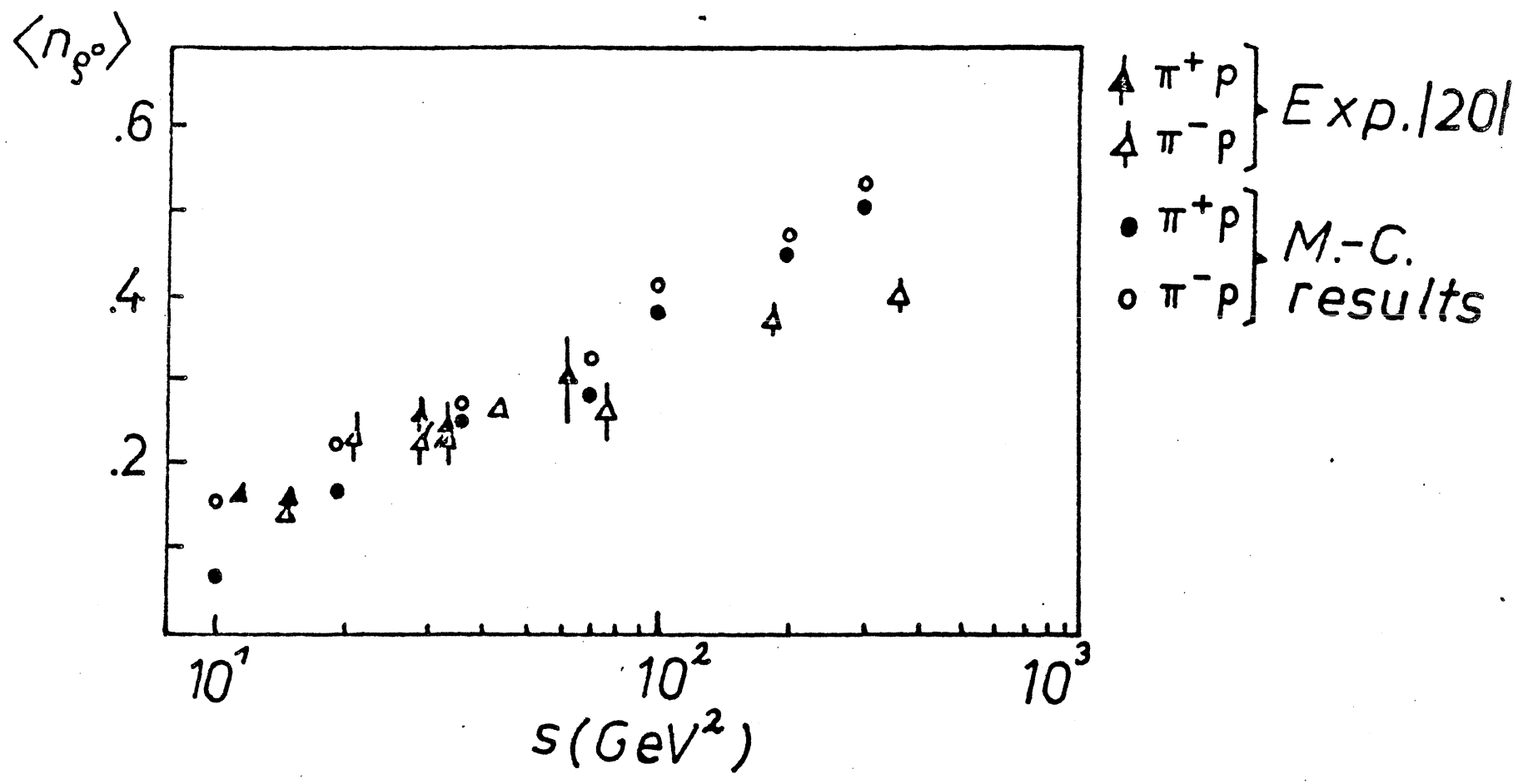

FIG.7 


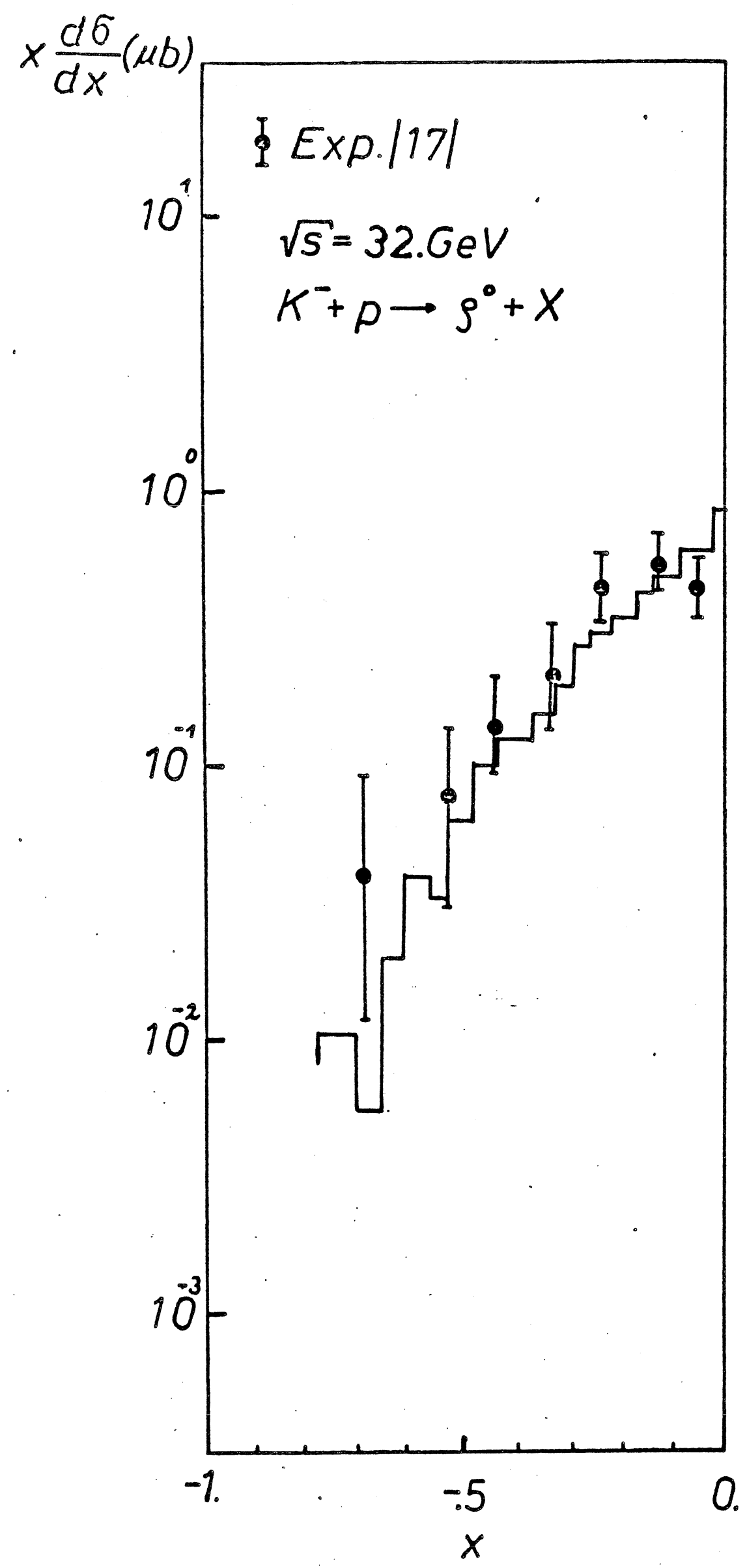

$F / G .8$ 


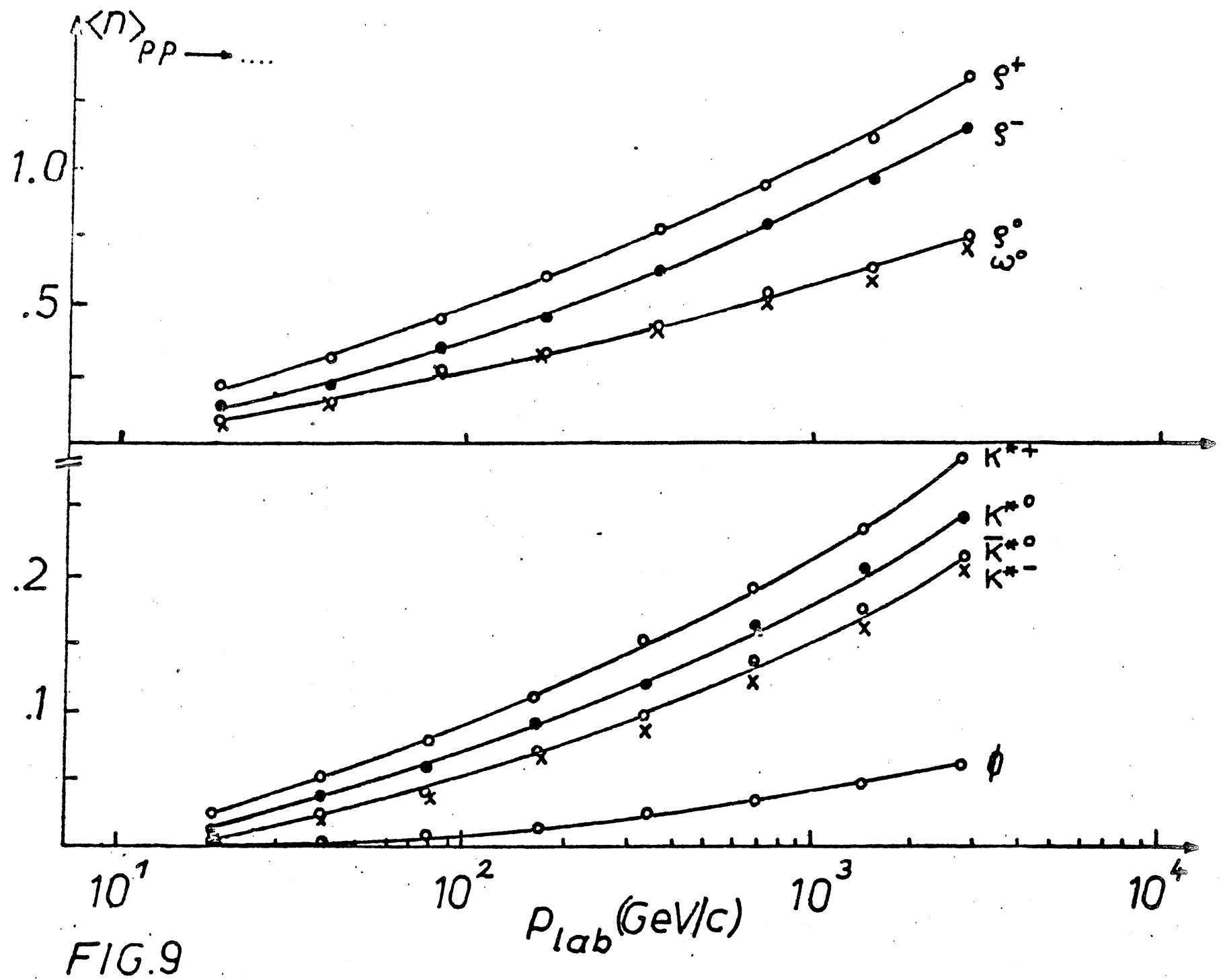


$\left\langle n_{F}\right\rangle$

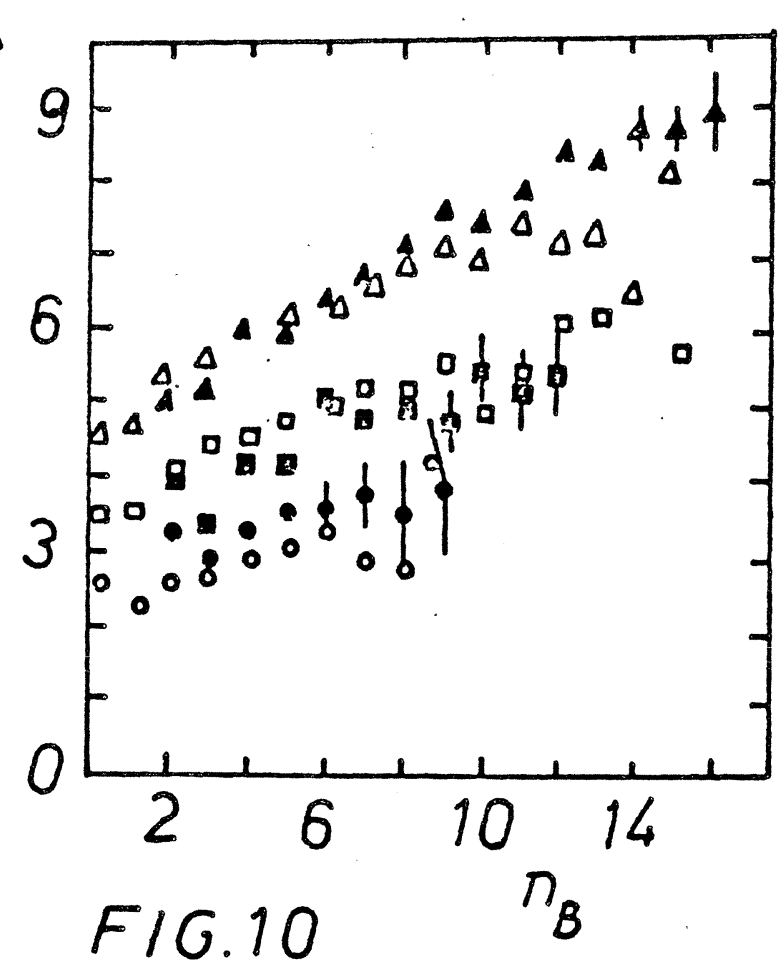

PP $\sqrt{5}(\mathrm{GeV})$

\begin{tabular}{l|l} 
Exp.1201 & M.-C. results \\
\hline 463 & 63.
\end{tabular} $463 . \Delta 63$.

申 $19.7 \square 30$.

+ $11.5 \mid \begin{array}{ll}0 & 11.5\end{array}$ $\left\langle n_{B}\right\rangle$

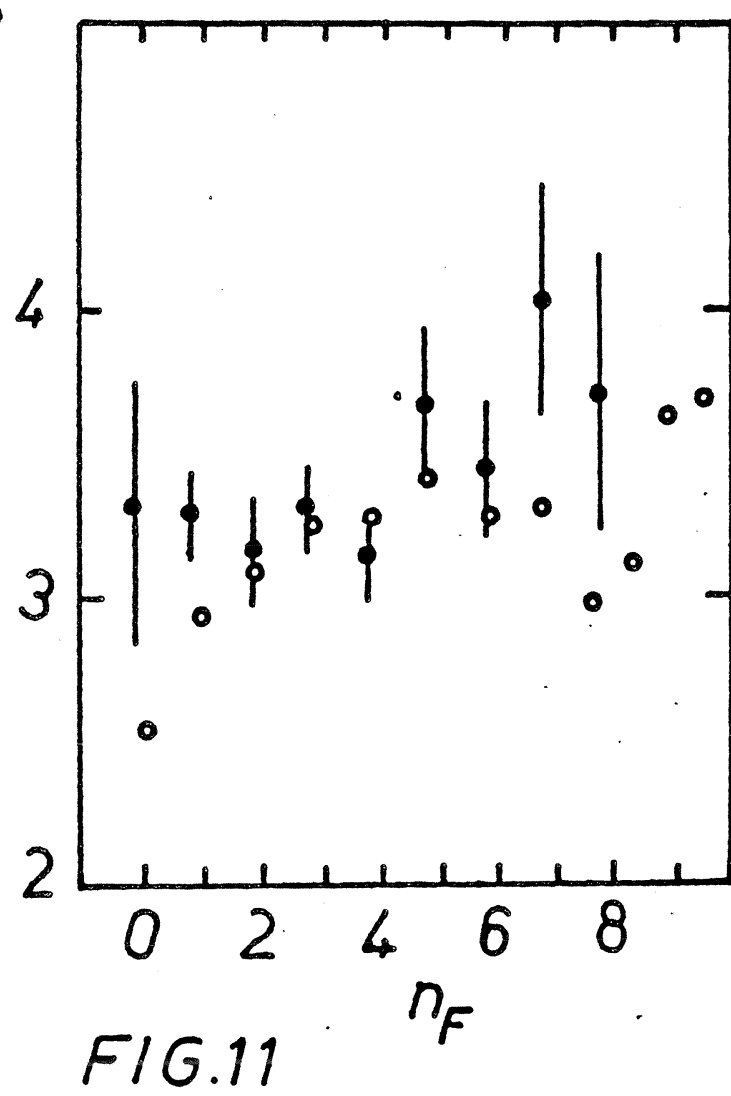

$\pi^{-} p \quad \sqrt{s}=13.7 \mathrm{GeV}$

| Exp. 120|

-M.-C.results 